\title{
Perfil de diversidade florística e padrão espacial de espécies arbóreas em florestas com ocorrência de bambu na Amazônia
}

\author{
Glória da Silva Almeida Leal $\bigcirc^{1 *}$ Fabrício Assis Leal $\bigodot^{1}$ Adriana da Silva Almeida da Cruz $\bigotimes^{1}$
}

${ }^{1}$ Universidade Federal do Acre, Rodovia BR 364, Km 04 - Distrito Industrial, CEP: 69920-900 78060-900, Rio Branco - AC, Brasil

\begin{abstract}
Original Article
*Corresponding author: gloriaalmeidaleal@gmail.com

Keywords:

Ecosystem conservation

Forest invetory

Foresty management

Palavras-chave:

Conservação de ecossistemas

Inventário florestal

Manejo florestal

Received in

RESUMO: No estado do Acre a área de reserva de bambu nativo é superior a qualquer outra no mundo, todavia esses ambientes ainda são raramente estudados. Logo, conhecer sua florística e estrutura é fator chave para o manejo e conservação desses ecossistemas. O objetivo deste trabalho foi avalia se a presença de bambu afeta a diversidade e a estrutura espacial de espécies florestais nativas. Para obtenção dos dados realizou-se um inventário em áreas de Floresta Aberta com Palmeira e Bambu (FAPB) e Floresta Aberta com Palmeira (FAP), localizadas na Resex Riozinho da Liberdade, no estado do Acre. Dos dados oriundos do inventário realizou-se a análise de cluster a fim de verificar a similaridade entre as áreas. Para comparar a diversidade florística foram avaliados o perfil de diversidade e o índice de Shannon. Já a estrutura espacial das espécies foi verificada pelo índice de Morisita. Os resultados apontaram maior diversidade florística para FAPB $\left(\mathrm{H}^{\prime}=3,83\right.$ e $\left.\mathrm{J}=0,87\right)$ em relação a $\mathrm{FAP}\left(\mathrm{H}^{\prime}=3,55\right.$ e $\mathrm{J}=$ 0,78 ), com diferença significativa ao nível de $5 \%$ de significância. A similaridade entre as áreas FAPB e FAP foi considerada baixa, apenas $28 \%$ das espécies são comuns as duas áreas. E quanto a estrutura espacial, as espécies apresentaram padrão de distribuição semelhante (agregado), tanto na FAPB quanto na FAP. Desta forma, conclui-se que o bambu não afetou a diversidade florística, tampouco a estrutura espacial das espécies.
\end{abstract}

2021/06/28

Accepted on

2021/10/06

Published in

2020/10/11

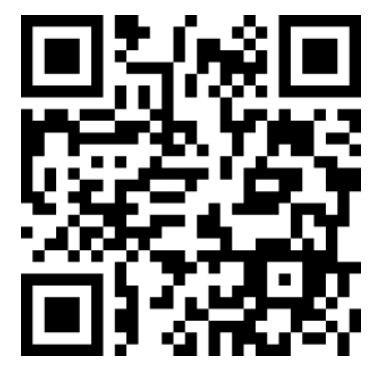

DOI:

http://dx.doi.org/10.34062/afs .v8i3.12678

\section{(cc) BY}

\section{Floristic diversity profile and spatial pattern of tree species in forests with bamboo occurrence in the Amazon}




\section{Introdução}

Devido a ampla distribuição geográfica do Bioma Amazônico, existem ainda muitas áreas inexploradas botanicamente e muitas novas espécies vêm sendo identificadas. No entanto, mesmo com inúmeros estudos já realizados, muito de sua flora ainda permanece desconhecida (Slik et al., 2015), especialmente em áreas com ocorrência de componentes arbóreos poucos estudados como o bambu, cuja abundância em florestas nativas tem sido motivo de muitas preocupações.

O bambu ocorre com importante presença em extensas áreas de florestas da Amazônia, e o estado do Acre é o local com a maior reserva de bambu nativo do mundo (Silva et al., 2019), onde ocupa $62 \%$ do território (Carmo et al., 2017). Como características, o bambu é um excelente colonizador, tem rápido crescimento e em função da sua estrutura clonal, também é um exímio competidor. Possui ainda, potencial de crescer indefinidamente por meio de ramificação horizontal dado a sua estratégia de crescimento vegetativo.

Essas características, por sua vez, podem limitar ou até mesmo impedir a regeneração de outras espécies nativas. Assim, pode-se esperar que áreas de florestas dominadas por bambu sejam menos diversificadas e apresentem composição florística distinta das florestas dominadas exclusivamente por palmeiras, por exemplo.

Contudo, mesmo com ampla distribuição, esses ambientes são raramente estudados e a compreensão de como a vegetação nativa está organizada e relacionada com a presença do bambu, ainda não é completa e é algo de muitas perguntas. Essa investigação, por meio de estudos científicos, é essencial para o manejo e conservação da biodiversidade nos ecossistemas onde o bambu está inserido. Assim sendo, o presente estudo objetivou avaliar se a ocorrência do bambu afeta a diversidade florística e a estrutura espacial de espécies florestais nativas.

\section{Material e métodos}

Este estudo foi realizado na Resex Riozinho da Liberdade (324.905 ha), situada entre os paralelos 7०30'00" a 9'00'00', de latitude sul e entre os meridianos $71^{\circ} 15^{\prime} 00^{\prime}$ ' a $72^{\circ} 30^{\prime} 00^{\prime}$ ' de longitude oeste, estado do Acre (Figura 1).

O clima da região é caracterizado como tropical, quente e úmido, com temperatura média anual de $24^{\circ} \mathrm{C}$ e precipitação anual superior a 2.000 mm. Os tipos de solos que predominam são Luvissolo, Argissolo, Cambissolo e Gleissolo, e quanto a cobertura florestal, $41,9 \%$ da Resex é coberta por Floresta Aberta com Palmeira associada a Floresta Densa e a Floresta Aberta com Bambu (Acre, 2010).

A coleta dos dados foi realizada em áreas de Floresta Aberta com Palmeira e Bambu (FAPB) e em áreas de Floresta Aberta com Palmeira (FAP). Foram instaladas 10 parcelas de dimensão $20 \times 50 \mathrm{~m}$, em cada uma das áreas. Todas as árvores no interior das parcelas, com circunferência a $1,30 \mathrm{~m}$ do solo (CAP), superior ou igual a $15 \mathrm{~cm}$, foram incluídas no estudo. Para as espécies não identificadas em campo, foram coletados material vegetal para posterior identificação botânica em laboratório. Os nomes científicos foram padronizados conforme a APG IV (2016)

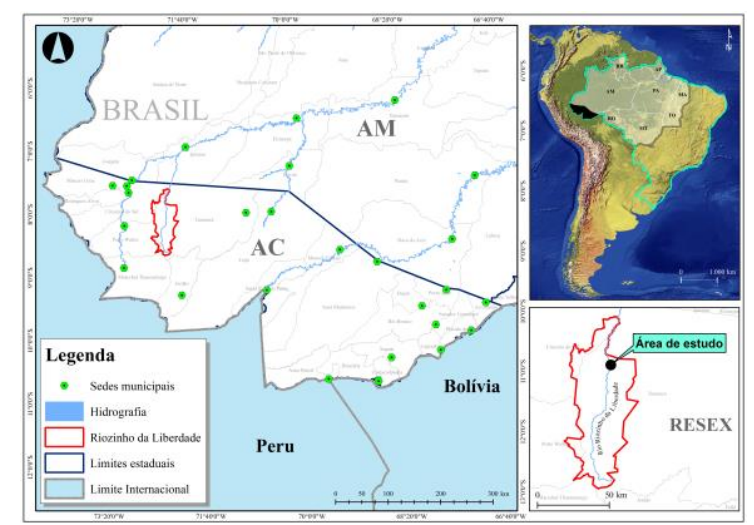

Figura 1. Localização da Resex Riozinho da Liberdade

A coleta dos dados foi realizada em áreas de Floresta Aberta com Palmeira e Bambu (FAPB) e em áreas de Floresta Aberta com Palmeira (FAP). Foram instaladas 10 parcelas de dimensão 20 x $50 \mathrm{~m}$, em cada uma das áreas. Todas as árvores no interior das parcelas, com circunferência a $1,30 \mathrm{~m}$ do solo (CAP), superior ou igual a $15 \mathrm{~cm}$, foram incluídas no estudo. Para as espécies não identificadas em campo, foram coletados material vegetal para posterior identificação botânica em laboratório. Os nomes científicos foram padronizados conforme a APG IV (2016).

A suficiência amostral do inventário foi avaliada pela curva de rarefação, com intervalos de confiança a $95 \%$ de probabilidade. As curvas de rarefação foram construídas segundo Gotelli e Colwell (2001), por meio do método de aleatorização, com 1.000 permutações, utilizando o programa EcoSim, versão 7.72 .

Para estudo da composição florística utilizouse a listagem da flora inventariada, a riqueza e a diversidade de espécies. E para as estimativas de similaridade florística entre as parcelas de FAP e FAPB empregou-se à análise de agrupamento (cluster), sendo adotado para a união entre os grupos o método das médias aritméticas de grupos não ponderados (UPGMA), com distância euclidiana. Foi utilizado para essa análise o índice de Jaccard (1991).

A consistência dos agrupamentos foi verificada pelo método bootstrap, com o uso do programa Bood v.1.3. Para medir o grau de ajuste entre a matriz de similaridade original e a matriz 
resultante da simplificação, proporcionada pelo método de agrupamento, calculou-se a correlação cofenética (rcof), conforme proposto por Bussab et al. (1990). O coeficiente de similaridade, a análise de agrupamento, a matriz cofenética e o valor da correlação cofenética foram calculados com o uso do programa computacional NTSYS v. 2.11X.

Para avaliação da diversidade, com a densidade de indivíduos registrada na menor amostra, foi calculada a riqueza de espécies, utilizando o número de espécies obtido por rarefação (S'rarefeita). Para medir a heterogeneidade de espécies foi utilizado o índice de Shannon (H'rarefeita), na base neperiana, por meio do programa EcoSim 7.72. Os valores do índice de diversidade (H') foram comparados por meio do teste t de Hutcheson, ao nível de 5\% de significância, para estas análises utilizou-se o programa PAST versão 4.02. Para medida de equabilidade empregouse o Índice Pielou, que varia de 0 (uniformidade mínima) a 1 (uniformidade máxima). Portanto, quanto mais próximo de 1, maior será a equabilidade. De forma adicional, para avaliar e comparar a diversidade florística entre as áreas, foram empregados os perfis de diversidade (Tóthmérész 1995), no qual utilizou-se a série exponencial de Rényi. Para as análises que se utilizou programas computacionais, adotou-se softwares que possuem acesso livre.

Por fim, para avaliar a estrutura espacial das espécies utilizou-se o índice de dispersão de Morisita (ID), sendo o ID aplicado apenas para as espécies comuns nas duas áreas. Esse índice, tem a vantagem de ser relativamente independente da média e do número de amostras. Quando ID $=1$, a distribuição é considerada ao acaso; quando ID $>1$, a distribuição é do tipo agregada e quando ID $<1$, a distribuição pode ser considerada regular. A significância do ID foi calculada pelo teste de aderência Qui-quadrado $\left(\chi^{2}\right)$, conforme critérios estabelecidos por (Costa et al. 2018).

Para as espécies que apresentaram diferenças no padrão de distribuição espacial, realizou-se a estratificação vertical, a fim de verificar o número de indivíduos por estrato.

\section{Resultados e discussão}

A tendência de estabilização da curva de rarefação (Figura 2), indicou que a adição de novas espécies não alteraria significativamente o número de espécies observadas (Kersten e Galvão, 2011). Portanto, a amostragem realizada na FAPB e FAP foi suficiente para representar a composição florística destes ambientes. Note ainda, que as tendências das curvas de rarefação mostraram padrões semelhantes entre FAP e FAPB, mas com riqueza de espécies superior na FAP.

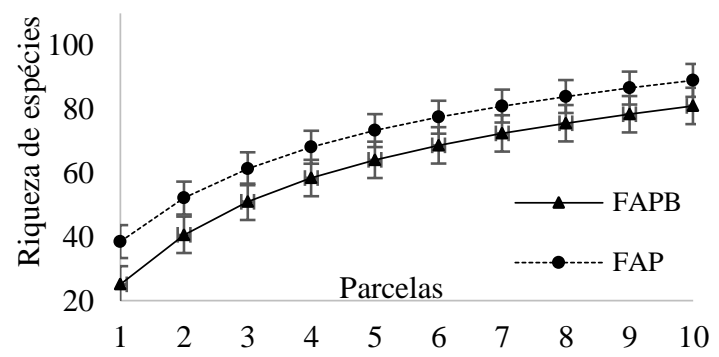

Figura 2. Curva de Rarefação das espécies amostradas para as áreas de Floresta Aberta com Palmeira e Bambu (FAPB) e para área de Floresta Aberta com Palmeira (FAP)

Na FAPB foram mensurados 4.010 colmos de bambu de Guadua weberbaueri Pilger e 647 indivíduos arbóreos pertencentes a 81 espécies, 76 gêneros e 29 famílias botânicas. Quanto na FAP, foram mensurados 1.152 indivíduos arbóreos, distribuídos em 89 espécies, 77 gêneros e 35 famílias botânicas (Tabela 1).

Tabela 1. Composição florística da vegetação arbórea nas áreas de Floresta Aberta com Palmeira e Bambu (FAPB) e Floresta Aberta com Palmeira (FAP).

\begin{tabular}{|c|c|c|c|}
\hline Família & Espécies & FAP $(n)$ & FAPB (n) \\
\hline Anacardiaceae & Spondias mombin L. & 2 & 4 \\
\hline Anacardiaceae & Anacardium giganteum W.Hancock ex Engl. & 1 & - \\
\hline Annonaceae & Annona cuspidata (Mart.) H.Rainer & 4 & - \\
\hline Annonaceae & Diclinanona calycina (Diels) R.E.Fr. & - & 2 \\
\hline Annonaceae & Duguetia surinamensis R.E.Fr. & 120 & 30 \\
\hline Annonaceae & Guatteria glauca Ruiz e Pav. & 20 & - \\
\hline Annonaceae & Malmea dielsiana R.E.Fr. & 2 & - \\
\hline Annonaceae & Oxandra espintana (Spruce ex Benth.) Baill. & - & 44 \\
\hline Apoccynaceae & Aspidosperma excelsum Benth. & - & 10 \\
\hline Apoccynaceae & Himatanthus sисииba Spruce ex Müll.Arg. & - & 6 \\
\hline Apocynaceae & Couma macrocarpa Barb.Rodr. & 27 & - \\
\hline Areacaceae & Attalea tessmannii Burret & 31 & 7 \\
\hline Areacaceae & Dictyocaryum ptarianum (Steyerm.) H.E.Moore. \& Steyerm. & 1 & - \\
\hline Areacaceae & Iriartea deltoidea Ruiz e Pav. & 4 & 20 \\
\hline Areacaceae & Oenocarpus bataua Mart. & 2 & 11 \\
\hline Areacaceae & Oenocarpus mapora H. Karst. & 7 & - \\
\hline
\end{tabular}




\begin{tabular}{|c|c|c|c|}
\hline Areacaceae & Socratea exorrhiza (Mart.) H. Wendl. & 5 & - \\
\hline Arecaceae & Astrocaryum aculeatum hort. ex H.Wendl. & 27 & 17 \\
\hline Arecaceae & Attalea phalerata Mart. ex Spreng. & 5 & 3 \\
\hline Arecaceae & Attlalea cf. butyracea (Mutis). & - & 10 \\
\hline Arecaceae & Bactris gasipaes Kunth. & - & 3 \\
\hline Arecaceae & Euterpe precatoria Mart & 36 & 16 \\
\hline Arecaceae & Mauritia flexuosa $\mathrm{L}$. f. & - & 1 \\
\hline Arecaceae & Socratea exorrhiza (Mart.) H.Wendl. & - & 20 \\
\hline Bignoniaceae & Handroanthus serratifolius (Vahl) S.Grose & - & 3 \\
\hline Bignoniaceae & Jacaranda copaia (Aubl.) D.Don. & - & 5 \\
\hline Bignoniaceae & Pithecoctenium crucigerum (L.) A.Gentry & 4 & 7 \\
\hline Bignoniaceae & Tabebuia insignis (Miq.) Sandwith & 3 & - \\
\hline Bixaceae & Cochlospermum orinocense (Kunth) Steud. & 3 & 4 \\
\hline Boraginaceae & Cordia alliodora (Ruiz e Pav.) Cham. ex A.DC. & - & 5 \\
\hline Boraginaceae & Cordia nodosa Lam. & - & 2 \\
\hline Caricaceae & Carica microcarpa (Jacq). & - & 4 \\
\hline Caricaceae & Carica microcarpa Jacq. & 1 & - \\
\hline Caryocaraceae & Caryocar glabrum (Aubl.) Pers. & - & 2 \\
\hline Chrysobalanaceae & Hirtella excelsa Standl. ex Prance & 2 & - \\
\hline Clusiaceae & Rheedia brasiliensis (C.Martius) P. \& T & 8 & 2 \\
\hline Elaeocarpaceae & Sloanea brevipes Benth. & 6 & - \\
\hline Elaeocarpaceae & Sloanea schomburgkii Benth. & 4 & - \\
\hline Euphorbiaceae & Caryodendron grandifolium (Müll.Arg.) Pax & - & 2 \\
\hline Euphorbiaceae & Dalechampia cissifolia Poepp. & 2 & 3 \\
\hline Euphorbiaceae & Hevea brasilliensis (Willd. ex Juss.) & 13 & 3 \\
\hline Euphorbiaceae & Hura crepitans L. & 11 & 2 \\
\hline Euphorbiaceae & Mabea piriri Aubl. & 21 & 2 \\
\hline Euphorbiaceae & Pausandra trianae (Muell. Arg.) Balll. & - & 2 \\
\hline Euphorbiaceae & Sapium glandulosum (L.) Morong & 2 & - \\
\hline Fabaceae & Copaifera krukovii (Dwyer) J.A.S. & 4 & - \\
\hline Fabaceae & Andira inermis (W.Wright) DC. & - & 4 \\
\hline Fabaceae & Apuleia leiocarpa (Vogel) J.F Macbr. & 4 & - \\
\hline Fabaceae & Bauhinia acreana Harms & 1 & 1 \\
\hline Fabaceae & Bowdichia martiusii Benth. & - & 3 \\
\hline Fabaceae & Cassia lucens Vogel & - & 11 \\
\hline Fabaceae & Cedrelinga cateniformis (Ducke) Ducke & - & 2 \\
\hline Fabaceae & Clitoria amazonum Mart. ex Benth. & 1 & 6 \\
\hline Fabaceae & Dalbergia riedelii (Benth.) Sandwith. & - & 7 \\
\hline Fabaceae & Dalbergia spruceana Benth & 3 & 10 \\
\hline Fabaceae & Dinizia excelsa Ducke & - & 6 \\
\hline Fabaceae & Dussia tessmannii Harms & 1 & 2 \\
\hline Fabaceae & Enterolobium schomburgkii (Benth.) Benth. & 6 & - \\
\hline Fabaceae & Erythrina amazonica Krukoff & - & 3 \\
\hline Fabaceae & Erythrina verna Vell. & 4 & - \\
\hline Fabaceae & Hymenaea courbaril L. & 7 & 3 \\
\hline Fabaceae & Hymenolobium nitidum Benth. & 3 & - \\
\hline Fabaceae & Inga acreana Harms & 83 & 37 \\
\hline Fabaceae & Myroxylon balsamum (L.) Harms & 1 & - \\
\hline Fabaceae & Parkia nitida Miq. & 3 & - \\
\hline Fabaceae & Platymiscium duckei Hub. & 14 & 5 \\
\hline Fabaceae & Vatairea sericea Ducke & 1 & - \\
\hline Lauraceae & Nectandra cissiflora Nees & 127 & - \\
\hline Lauraceae & Mezilaurus itauba (Meisn.) Taub. ex Mez & 1 & - \\
\hline Lauraceae & Nectandra amazonum Nees & 31 & 9 \\
\hline Lauraceae & Nectandra cuspidata Nees & - & 7 \\
\hline Lauraceae & Nectandra maynensis Mez. & 1 & - \\
\hline Lecythidaceae & Eschweilera coriacea DC. S.A. Mori. & 61 & 12 \\
\hline Malvaceae & Gossypium barbadense L., n. c. & - & 2 \\
\hline Malvaceae & Ceiba pentandra (L.) Gaertn. & 7 & 11 \\
\hline
\end{tabular}




\begin{tabular}{|c|c|c|c|}
\hline Malvaceae & Guazuma ulmifolia Lam. & - & 2 \\
\hline Malvaceae & Heliocarpus americanos $\mathrm{L}$. & - & 44 \\
\hline Malvaceae & Matisia cordata Kunth & 20 & 4 \\
\hline Malvaceae & Sterculia chicomendesii E.L.Taylor & 10 & - \\
\hline Malvaceae & Theobroma cacao L. & 6 & - \\
\hline Malvaceae & Theobroma grandiflorum (Willd. ex Spreng.) K.Schum. & - & 2 \\
\hline Meliaceae & Cedrela odorata L. & - & 3 \\
\hline Meliaceae & Guarea acreana C.DC. & 2 & 7 \\
\hline Meliaceae & Guarea kunthiana A.Juss. & 2 & - \\
\hline Meliaceae & Guarea macrophylla Vahl & 5 & - \\
\hline Moraceae & Brosimum lactescens (S.Moore) C.C.Berg. & - & 6 \\
\hline Moraceae & Castilla ullei Warb. & 1 & 5 \\
\hline Moraceae & Clarisia racemosa Ruiz e Pav & 6 & 7 \\
\hline Moraceae & Ficus insipida Wild & 4 & 17 \\
\hline Moraceae & Ficus lauretana Vázq. Avila & 8 & - \\
\hline Moraceae & Ficus maxima Mill. & 5 & - \\
\hline Moraceae & Maclura tinctoria (L.) D. Don ex Steud. & 3 & 7 \\
\hline Moraceae & Naucleopsis caloneura (Huber) Ducke & 17 & 5 \\
\hline Moraceae & Pseudolmedia laevis (Ruiz e Pav.) J.F.Macbr. & 28 & 10 \\
\hline Myristicaceae & Virola pavonis (A.DC.) A.C.Sm & 48 & 29 \\
\hline Myristicaceae & Virola sebifera Aubl. & 13 & - \\
\hline Myrtaceae & Eugenia cupulata Amshoff & 1 & - \\
\hline Myrtaceae & Eugenia pseudopsidium Jacq. & 1 & - \\
\hline Myrtaceae & Psidium guajava L. & - & 1 \\
\hline Nyctaginaceae & Neea madeirana Standl. & 26 & 6 \\
\hline Olacaceae & Minquartia guianensis Aubl. & 3 & - \\
\hline Phyllanthaceae & Hyeronima laxiflora Muell. Arg. & 1 & - \\
\hline Phytolacaceae & Gallesia gorasema Moq. & 1 & - \\
\hline Piperaceae & Piper cf. aduncum L. & - & 1 \\
\hline Piperaceae & Piper hispidinervium C.DC. & 1 & - \\
\hline Poaceae & Guadua weberbaueri Pilger & - & 4.010 \\
\hline Polygonaceae & Triplaris surinamensis Cham. & - & 3 \\
\hline Polygonaceae & Triplaris weigeltiana (Rchb.) Kuntze & 10 & - \\
\hline Rhamnaceae & Ziziphus cinnamomum Triana e Planch. & 2 & - \\
\hline Rubiaceae & Alibertia edulis (Rich.) A.Rich. & 1 & - \\
\hline Rubiaceae & Calycophyllum acreanum Ducke & 7 & 3 \\
\hline Rubiaceae & Calycophyllum spruceanum (Benth.) K.Schum. & 6 & 1 \\
\hline Rubiaceae & Genipa americana $\mathrm{L}$. & 3 & 7 \\
\hline Rubiaceae & Sickingia tinctoria (Schult.) K.Schum. & 4 & - \\
\hline Rutaceae & Pilocarpus peruvianus (J.F.Macbr.) Kaastra & - & 2 \\
\hline Rutaceae & Zanthoxylum sprucei Engl. & 5 & 3 \\
\hline Salicaceae & Banara nitida Spruce ex Benth. & 1 & - \\
\hline Sapindaceae & Paullinia alata (Ruiz e Pav.) Don & - & 1 \\
\hline Sapindaceae & Talisia cf. marcophylla Radlk & - & 1 \\
\hline Sapotaceae & Manilkara inundata Ducke & 3 & - \\
\hline Sapotaceae & Chrysophyllum lucentifolium Cronquist & 2 & - \\
\hline Sapotaceae & Manilkara bidentata (A.DC.) A.Chev. & 5 & 1 \\
\hline Sapotaceae & Pouteria gardneri (Mart. \& Miq.) Baehni & 1 & - \\
\hline Sapotaceae & Pouteria macrophylla (Lam.) Eyma & - & 51 \\
\hline Sapotaceae & Pouteria nudipetala T.D.Penn. & 114 & - \\
\hline Simaroubaceae & Simarouba amara Aubl. & 7 & - \\
\hline Urticaceae & Cecropia membranacea Trécul & - & 12 \\
\hline Urticaceae & Pourouma guianensis Aubl. & - & 13 \\
\hline Violaceae & Rinorea longistipulata Hekking & 31 & - \\
\hline Total Geral & & 1152 & 4657 \\
\hline
\end{tabular}

*n = número de indivíduos por espécie.

Foi observado que a FAPB, em relação a FAP, apresentou menor densidade de indivíduos e menor riqueza de espécies, resultados equivalentes também foram observados nos trabalhos de Felker et al. (2017; 2018). Ressaltamos, que esse impacto negativo pode estar relacionado ao caráter 
potencialmente invasor do bambu (Buziquia et al., 2019). Segundo a pesquisa de D’Oliveira et al. (2013), se tipologias com ocorrência do bambu sofrerem algum tipo de perturbação, isso implica no aumento na densidade de colmos de bambu e consequentemente redução na densidade e riqueza de espécies.

Quanto a riqueza de espécies, na FAPB as famílias com maior representatividade foram: Fabaceae (14), Arecaceae (9), Moraceae (7), Malvaceae, Euphorbiaceae, (6), e representaram 52\% dos indivíduos. Na FAP, destacaram-se as famílias: Fabaceae (14), Arecaceae (9), Moraceae (7) Sapotaceae (6), e Euphorbiaceae e Rubiaceae (5), com $45 \%$ dos indivíduos inventariados (Tabela 1 ).

Nota-se, que as famílias mais diversificadas foram comuns tanto na FAPB quanto na FAP, pois estas são comumente amostradas em florestas da Amazônia. Confirmam esses resultados o estudo de Demarchi et al. (2018), onde igualmente destacaram as famílias Moraceae, Malvaceae, Euphorbiaceae, Sapotaceae, como as mais representativas na região Amazônica, ressaltando maior riqueza observada à Fabaceae.

No que tange as espécies de maior densidade, das vinte espécies predominantes, observou-se que cinco ocorrem tanto na FAPB quanto na FAP (Inga acreana, Duguetia surinamensis, Eschweilera coriacea, Euterpe precatoria, Virola pavonis), sendo essas espécies comuns em diferentes tipologias florestais no Acre. De modo geral, quanto a riqueza de espécies observada, os resultados obtidos por Rockwell et al. (2015) e Freitas et al. (2018), também apresentaram resultados similar a esse estudo.

Destacamos, que dentre as espécies supracitadas e que são comuns às duas áreas deste estudo, as espécies Eschweilera coriacea e Euterpe precatoria, de acordo com a pesquisa de Steege et al. (2013), estão entre as espécies hiperdominantes ao longo da Bacia Amazônica. Segundo Levis et al. (2017), pode ser resultado da influência da ação humana no passado, como a domesticação de plantas pelos povos pré-colombianos que resultou na atual composição e estrutura das florestas da Amazônia.

A similaridade florística entre FAPB e FAP foi baixa $(\mathrm{J}=0,28)$, de forma complementar, a análise de agrupamento (Figura 3), indicou a formação de dois grupos distintos (FAPB e FAP) o que confirma a forte diferença na composição florística entre as áreas, inferindo que a presença do bambu altera a composição florestal. Ressaltamos, que a estabilidade do agrupamento foi de $99 \%$ para o grupo da FAPB e de $73 \%$ para o grupo da FAP, o que indicou consistência dos agrupamentos. A correlação cofenética de 0,95 , indica que houve excelente representação das matrizes de dissimilaridade na forma de dendrograma.

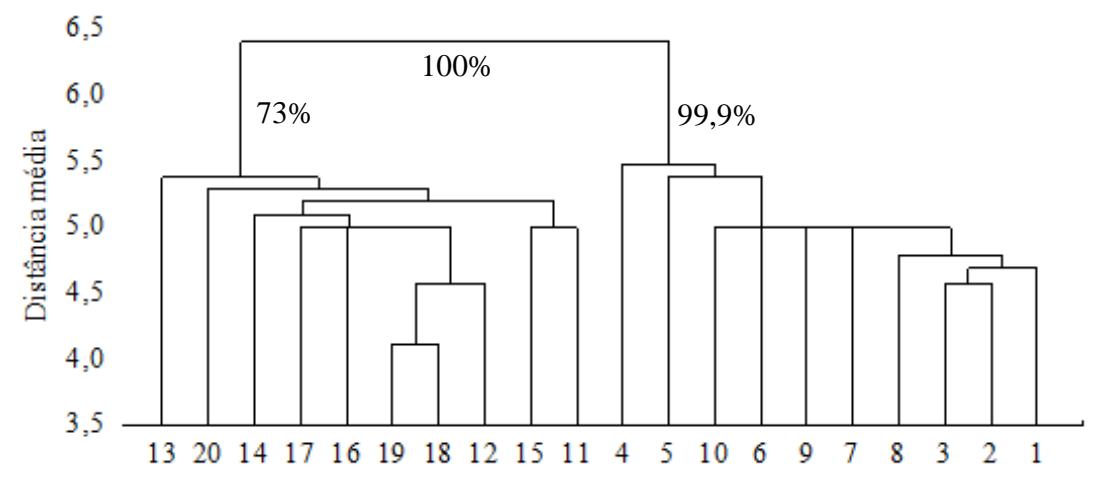

Figura 3. Análise de agrupamento de espécies entre parcelas para Floresta Aberta com Palmeira e Bambu (FAPB, parcelas 1 a 10) e Floresta Aberta com Palmeira (FAP, parcelas 11 a 20)

As curvas de rarefação para comparação de riqueza com intensidade amostral padronizada (Figura 4A), apontaram que FAPB apresentou maior riqueza de espécies que a FAP, fato evidenciado pelo ponto exato de rarefação, que é o ponto onde a riqueza foi dada com o mesmo número de indivíduos $(n=600)$, para as duas áreas em estudo. Ademais, os perfis de diversidade de espécies revelaram notável variação entre FAPB e FAP. Note, que as curvas se cruzaram no intervalo de confiança quando $\alpha>0,28$, o que inferiu semelhança na riqueza e na equabilidade (Figura 4B), e para qualquer valor do parâmetro alfa até $\alpha<0,32$, independente do índice de diversidade utilizado, a comunidade FAPB foi mais diversa que a FAP. 

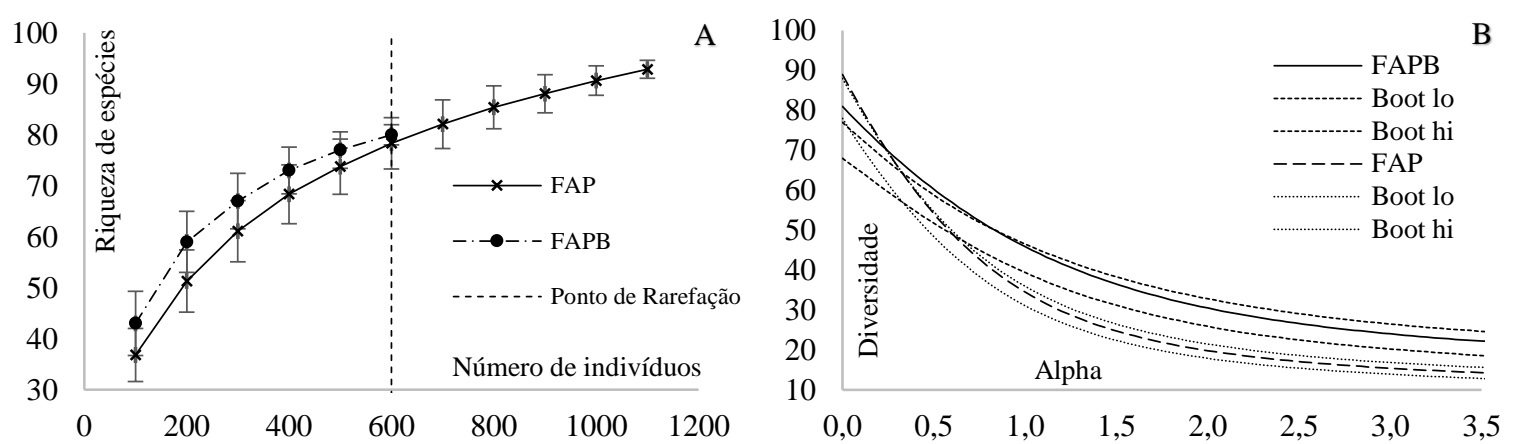

Figura 4. Curvas de rarefação de espécies, para comparação de riqueza com intensidade amostral padronizada (A); perfis de Diversidade de espécies lenhosas (B), para as áreas de Floresta Aberta com Palmeira e Bambu (FAPB) e para área de Floresta Aberta com Palmeira (FAP)

Os valores obtidos pelo índice de Shannon indicam maior diversidade para FAPB $\left(\mathrm{H}^{\prime}=3,84 \mathrm{e}\right.$ $\mathrm{J}=0,87$ ), que FAP ( $\mathrm{H}^{\prime}=3,55$ e $\mathrm{J}=0,78$ ), ao nível de $5 \%$ de significância $(\mathrm{p}<0,000)$. Note, que as duas áreas apresentaram equabilidade (J) próximo a 1, e valores de $\mathrm{J}$ que variaram entre 0,75 e 0,92 foram considerados satisfatórios (Batista et al., 2015). Posto isso, infere-se que há uniformidade da abundância dos indivíduos entre as espécies existentes, que por sua vez, contribui para a elevar da diversidade florística da comunidade vegetal.

Corroboram com os resultados de diversidade supracitados, as pesquisas de Freitas et al. (2018) e Oliveira et al. (2019), que encontraram valores de Shannon variando de H'=3,87 a 4,03. Além disso, os valores observados nas florestas objeto desse estudo estão próximos ao esperado para florestas altamente diversificada, cujo H' apresentam-se entre 3,83 a 5,85 (Knight, 1975). Estes resultados mostraram que o bambu não afetou de forma drástica a diversidade florística, quando comparado a outras áreas sem a ocorrência de bambu. Tal fato, segundo Yuen et al. (2017), foi contatado também ao verificar que a presença de algumas espécies de plantas estava relacionada a ocorrência do bambu.

Quanto a distribuição espacial (Tabela 2), é possível notar que o valor do $\chi^{2}$ calculado, foi maior que o valor do $\chi^{2}$ tabelado, ao nível de $5 \%$ de significância, para todas as espécies, tanto na FAPB quanto na FAP. Tal fato denota distribuição agregada, exceto para a espécie Astrocaryum aculeatum, que apresentou padrão espacial agregado na FAPB e aleatório na FAP.

Tabela 2. Distribuição espacial das espécies comuns nas áreas FAPB e FAP.

\begin{tabular}{lrrrrr}
\hline \multicolumn{1}{c}{ Espécies } & Área & IM & $\chi^{2}$ calc. & $\chi^{2}$ tab. & ID $\left(\chi^{2}\right)$ \\
\hline \multirow{2}{*}{ Attalea phalerata } & FAPB & 1,01 & 7,00 & 3,33 & agregado \\
Attalea tessmannii & FAP & 0,01 & 5,00 & agregado \\
& FAPB & 2,86 & 7,00 & agregado \\
Astrocaryum aculeatum & FAP & 0,95 & 7,39 & agregado \\
Bauhinia acreana & FAPB & 1,25 & 13,00 & agregado \\
Calycophyllum acreanum & FAP & 0,77 & 3,00 & aleatório \\
& FAPB & 0,01 & 9,00 & agregado \\
Calycophyllum spruceanum & FAP & 0,01 & 9,00 & agregado \\
Castilla ullei & FAPB & 0,01 & 7,00 & agregado \\
& FAP & 1,90 & 14,43 & agregado
\end{tabular}


Leal et al.

\begin{tabular}{|c|c|c|c|c|}
\hline Ceiba pentandra & $\begin{array}{r}\text { FAPB } \\
\text { FAP }\end{array}$ & $\begin{array}{l}5,64 \\
0,48\end{array}$ & $\begin{array}{r}55,36 \\
5,86\end{array}$ & $\begin{array}{l}\text { agregado } \\
\text { agregado }\end{array}$ \\
\hline \multirow{2}{*}{ Clarisia racemosa } & FAPB & 3,33 & 23,00 & agregado \\
\hline & FAP & 0,67 & 7,33 & agregado \\
\hline \multirow{2}{*}{ Clitoria amazonum } & FAPB & 2,00 & 14,00 & agregado \\
\hline & FAP & 0,01 & 9,00 & agregado \\
\hline \multirow{2}{*}{ Cochlospermum orinocense } & FAPB & 10,00 & 36,00 & agregado \\
\hline & FAP & 0,01 & 7,00 & agregado \\
\hline \multirow{2}{*}{ Dalbergia spruceana } & FAPB & 0,48 & 5,86 & agregado \\
\hline & FAP & 0,01 & 14,43 & agregado \\
\hline \multirow{2}{*}{ Dalechampia cissifolia } & FAPB & 3,33 & 13,67 & agregado \\
\hline & FAP & 0,01 & 8,00 & agregado \\
\hline \multirow{2}{*}{ Duguetia surinamensis } & FAPB & 2,69 & 58,00 & agregado \\
\hline & FAP & 0,98 & 6,50 & agregado \\
\hline \multirow{2}{*}{ Dussia tessmannii } & FAPB & 0,01 & 9,00 & agregado \\
\hline & FAP & 0,01 & 9,00 & agregado \\
\hline \multirow{2}{*}{ Euterpe precatoria } & FAPB & 1,67 & 19,00 & agregado \\
\hline & FAP & 0,87 & 4,56 & agregado \\
\hline \multirow{2}{*}{ Eschweilera coriacea } & FAPB & 10,00 & 99,00 & agregado \\
\hline & FAP & 0,98 & 7,69 & agregado \\
\hline \multirow{2}{*}{ Ficus insipida } & FAPB & 1,67 & 19,00 & agregado \\
\hline & FAP & 1,67 & 11,00 & agregado \\
\hline \multirow{2}{*}{ Genipa americana } & FAPB & 5,24 & 34,43 & agregado \\
\hline & FAP & 0,01 & 7,00 & agregado \\
\hline \multirow{2}{*}{ Guarea acreana } & FAPB & 0,95 & 8,71 & agregado \\
\hline & FAP & 0,01 & 8,00 & agregado \\
\hline \multirow{2}{*}{ Hevea brasilliensis } & FAPB & 10,00 & 27,00 & agregado \\
\hline & FAP & 1,03 & 9,31 & agregado \\
\hline \multirow{2}{*}{ Hura crepitans } & FAPB & 0,01 & 9,00 & agregado \\
\hline & FAP & 0,55 & 4,45 & agregado \\
\hline \multirow{2}{*}{ Hymenaea courbaril } & FAPB & 0,01 & 9,00 & agregado \\
\hline & FAP & 0,95 & 8,71 & agregado \\
\hline \multirow{2}{*}{ Inga acreana } & FAPB & 2,46 & 61,65 & agregado \\
\hline & FAP & 0,95 & 4,83 & agregado \\
\hline \multirow{2}{*}{ Iriartea deltoidea } & FAPB & 2,42 & 36,00 & agregado \\
\hline & FAP & 1,67 & 11,00 & agregado \\
\hline \multirow[b]{2}{*}{ Mabea piriri } & FAPB & 10,00 & 18,00 & agregado \\
\hline & FAP & 0,81 & 5,19 & agregado \\
\hline
\end{tabular}


FAPB $\quad 1,43 \quad 11,57 \quad$ agregado

Maclura tinctoria

\begin{tabular}{|c|c|c|c|c|}
\hline & FAP & 0,01 & 7,00 & agregado \\
\hline \multirow{2}{*}{ Manilkara bidentata. } & FAPB & 1,01 & 9,00 & agregado \\
\hline & FAP & 3,00 & 17,00 & agregado \\
\hline \multirow{2}{*}{ Matisia cordata } & FAPB & 1,67 & 11,00 & agregado \\
\hline & FAP & 1,37 & 16,00 & agregado \\
\hline \multirow{2}{*}{ Naucleopsis caloneura } & FAPB & 6,00 & 29,00 & agregado \\
\hline & FAP & 0,66 & 3,59 & agregado \\
\hline \multirow{2}{*}{ Nectandra amazonum } & FAPB & 1,79 & 14,50 & agregado \\
\hline & FAP & 1,27 & 17,06 & agregado \\
\hline \multirow{2}{*}{ Neea madeirana } & FAPB & 2,00 & 14,00 & agregado \\
\hline & FAP & 0,92 & 7,08 & agregado \\
\hline \multirow{2}{*}{ Oenocarpus bataua } & FAPB & 2,89 & 26,00 & agregado \\
\hline & FAP & 0,01 & 9,00 & agregado \\
\hline \multirow{2}{*}{ Platymiscium duckei } & FAPB & 0,01 & 11,00 & agregado \\
\hline & FAP & 1,10 & 10,29 & agregado \\
\hline \multirow{2}{*}{ Pithecoctenium crucigerum } & FAPB & 2,86 & 20,14 & agregado \\
\hline & FAP & 0,01 & 6,00 & agregado \\
\hline \multirow{2}{*}{ Pseudolmedia laevis } & FAPB & 3,11 & 28,00 & agregado \\
\hline & FAP & 0,82 & 4,14 & agregado \\
\hline \multirow{2}{*}{ Rheedia brasiliensis } & FAPB & 1,01 & 9,00 & agregado \\
\hline & FAP & 1,07 & 9,50 & agregado \\
\hline \multirow{2}{*}{ Spondias mombin } & FAPB & 1,67 & 11,00 & agregado \\
\hline & FAP & 0,01 & 8,00 & agregado \\
\hline \multirow{2}{*}{ Virola pavonis } & FAPB & 2,24 & 43,76 & agregado \\
\hline & FAP & 0,98 & 8,25 & agregado \\
\hline \multirow{2}{*}{ Zanthoxylum sprucei } & FAPB & 3,33 & 13,67 & agregado \\
\hline & FAP & 1,00 & 9,00 & agregado \\
\hline
\end{tabular}

Onde: IM = valor do índice de Morisita; ID (IM) = índice de dispersão de Morisita; $\chi^{2}$ calc. = valor do qui-quadrado calculado; $\chi^{2}$ tab. = valor do qui-quadrado tabelado; ID $\left(\chi^{2}\right)=$ índice de dispersão baseado no teste do qui-quadrado.

Rockwell et al. (2017), ratifica ser comum o padrão de distribuição agregado para espécies arbóreas, pois essa tendência de agrupamento decorre: das formas de reprodução, da presença de distúrbios, de fatores ambientais que limitam sua distribuição, pelas complexas interações especificas e interespecíficas entre membros da comunidade vegetal ou até mesmo pela baixa competição intraespecífica na população e dispersão restrita de sementes próximas à planta mãe (Song et al., 2017; Varella et al., 2018).
Quanto a espécie Astrocaryum aculeatum, que apresentou padrão espacial aleatório na FAP e padrão agregado na FAPB, isso pode ser explicado pela estrutura dessa espécie em cada uma das comunidades onde ela está inserida. Na FAP, a maior concentração de indivíduos ocorreu no estrato inferior $(\mathrm{n}=15)$, apresentando-se de forma decrescente nos estratos médio $(\mathrm{n}=11)$ e superior $(\mathrm{n}$ =1). Quanto a FAPB, a densidade desta espécie concentrou-se no estrato inferior $(\mathrm{n}=16)$, e representou $94,1 \%$ do total, e não foi registrado nenhum indivíduo no estrato médio e estrato 
superior $\operatorname{com} n=1$. Isso evidenciou que a população da Astrocaryum aculeatum na FAPB é jovem e com distúrbios de estabelecimento. Junior et al. (2017), corroboram com estes resultados, onde indivíduos jovens ou menores, geralmente, apresentam padrões agregados; e os indivíduos adultos ou maiores, geralmente apresentam padrões aleatórios.

\section{Conclusão}

A Floresta Aberta com Palmeira e bambu apresentou menor densidade de indivíduos e menor riqueza de espécies. Todavia, a presença do bambu não afetou a diversidade florística e o padrão de distribuição espacial das espécies, pois tanto a FAP quanto a FAPB apresentaram elevados índices de Shannon e padrão de distribuição espacial de espécies semelhantes.

\section{Referências}

Acre Governo do Estado do Acre (2010) Zoneamento Ecológico-Econômico do Acre, Fase II: documento Síntese - Escala 1:250.000. Rio Branco: SEMA, 354 p.

Batista APB, Aparício WCS, Aparício OS, Santos VS, Lima RB, Mello JM (2015) Caracterização estrutural em uma floresta de terra firme no estado do Amapá, Brasil. Pesquisa Florestal Brasileira, 35 (81):21-33. doi: 10.4336/2015.pfb.35.81.689

Bussab WO, Miazaki ES, Andrade DF (1990) Introdução à análise de agrupamentos. São Paulo: Associação Brasileira de Estatística, 105p.

Buziquia ST, Lopes PVF, Almeida AK, Almeida, IK (2019) Impacts of bamboo spereading: a review. Biodiversity and Conservation, 28(14): 3695-3711. doi.org/10.1007/s10531-019-01875-9

Carmo LFZ, Amaral EF, Bardales NG (2017) Ocorrência, biomassa, perdas e exploração de bambu em florestas da Amazônia no Acre, Brasil. In: DRUMOND, P. M.; WIEDMAN, G. (Org.). Bambus no Brasil: da biologia à tecnologia. Rio de Janeiro - RJ: Instituto Ciência Hoje, v.1, 160p.

Costa DL, Santos MF, Bezerra TG, Ribeiro RBS, Vasconcellos RV, Melo LO, Cimenes LC, Coelho AA (2018) Estrutura e distribuição espacial de Symphonia globulifera L. f. em floresta de várzea baixa, Afuá-PA. Advances in Forestry Science, 5:275-

281,https://pdfs.semanticscholar.org/40f5/6807c790 94570e8d93ee4e8038f881836b77.pdf

Dantas AR, Marangon LC, Guedes M C, Feliciano, ALP, Lira-Guedes AC (2017) Spatial distribution of a population of Pentaclethra macroloba (Willd.) Kuntze in a floodplain forest of the Amazon Estuary. Revista Árvore, 41:1-11. doi: http://dx.doi.org/10.1590/1806-

90882017000400006

Demarchi LO, Scudeller VV, Moura LC, DiasTerceiro RG, Lopes A, Wittmann FK, Piedade MTF (2018) Composição florística, estrutura e relação solo-vegetação em três áreas de campinarana na Amazônia central. Acta Amazonica, 48(1):46. doi.org/10.1590/1809-4392201603523

D'Oliveira MVN, Guarino ES, Oliveira LC, Ribas LA, Acuña HÁ (2013). Can forest management be sustainable in a bamboo dominated forest? A 12year study of forest dynamics in western Amazon. Forest Ecology and Management. 310:672-679. doi.org/10.1016/j.foreco.2013.09.008

Felker RM, Rovedder APM, Longhi SJ, Araujo EF, Stefanello MM, Junior JCC, Procknow D, Hummel RB, Piaia BB, Camargo B, Silva MPKL, Toso LD (2018) Regeneração natural em área sob domínio de bambu, no sul do Brasil. Revista de Ciências Agrárias. doi.org/10.19084/RCA17100

41(1):82-92.

Felker RM, Rovedder APM, Longhi SJ, Araujo EF, Stefanello MM, Pecatti A (2017) Impact of Bambusa tuldoides Munro (Poaceae) on forest regeneration. Cerne, 23(2): 275-282, doi.org/10.1590/01047760201723022297

Freitas JL, Silva RBL, Junior FOC, Cantuária PC, Medeiros TDS, Santos ES (2018) Composição florística arbórea em reserva extrativista no Amapá. Revista em Agronegócio e Meio Ambiente, 11(1):277-300. doi.org/10.17765/21769168.2018v11n1p277-300

Gotelli NJ, Colwell RK (2001) Quantifying biodiversity: procedures and pitfalls in the measurement and comparison of species richness. Ecology Letters, 4:379-391. doi.org/10.1046/j.14610248.2001.00230.x

Junior MSM, Mota SL, Machado EJM, Pereira IM (2017) Distribuição espacial de Eremanthus incanus (Less.) Less. (Asteraceae) em duas áreas com diferentes níveis de conservação. Revista Brasileira de Biociências, 15(1): 27-31. doi.org/10.5380/abclima.v21i0.46432

Kersten, R.A, Galvão F (2011) Suficiência amostral em inventários florísticos e fitossociológicos. In: FELFILI, J. M. et al. Fitossociologia no Brasil: métodos e estudos de casos. Minhas Gerais: UFV. p. 156-173.

Knight DH (1975) A phytosociological analysis of species-rich tropical forest on Barro Colorado 
Island, Panama. Ecological Monographs, 5(3):.259284. doi: $10.2307 / 1942424$

Levis C et al (2017) Persistent effects of preColumbian plant domestication on Amazonian forest composition. Science, v.355, 6328: 925-931. doi: 10.1126/science.aal0157

Oliveira EKB, Rezende AV, Freitas LJM, Júnior LSM, Barros QS, Costa LS (2019) Monitoramento da estrutura e caracterização ecológica em floresta tropical manejada na Amazônia Brasileira. Revista Brasileira de Ciências Agrárias. 14(4):6867. doi:10.5039/agraria.v14i4a6867

Rockwell CA, Guariguata MR, Menton M, Quispe E A, Quaedvlieg J (2017) Warrem-Thomas, E., Silva, H. F., ... Sala, J. J. Y. Spatial distribution of Bertholletia excelsa in selectively logged forests of the Peruvian Amazon. Journal of Tropical Ecology, 33(2):114-127.

doi.org/10.1017/S0266467416000614

Rockwell CA, Kainer KA (2015) Local and scientific perspectives on the bamboo-dominated forest in Acre, Brazil: a complementary knowledge base for multiple-use forest management. International Forestry Review, 17(1):51-64. doi.org/10.1505/146554815814668945

Silva SMS, Pereira JES, Silva WC (2019) Conservação e diversidade de bambu Guadua no Acre. $1^{\circ}$ ed. Rio Branco- AC: Instituto Federal do Acre. $83 \mathrm{p}$.
Slik JW, Rodríguez VA, Aiba S, Loayza PA, Alves LF, Ashton P, Balvanera P (2015) An estimate of the number of tropical tree species. Proceedings of the National Academy of Sciences, 112(4):7472-7477. doi.org/10.1073/pnas.1423147112

Song H, Xu Y, Hao J, Zhao B, Guo D, Shao H (2017) Investigating distribution pattern of species in a warm-temperate conifer-broadleaved-mixed forest in China for sustainably utilizing forest and soils. Science of The Total Environment, 578:81-89. doi.org/10.1016/j.scitotenv.2016.07.218

Steege HT et al (2013) Hyperdominance in the Amazonian Tree Flora. Science, 342(6156):325-337. doi: 10.1126/science.1243092

Tóthmérész B (1995) Comparison of different methods for diversity ordering. Journal of Vegetation Science, 6(2):283-290, doi.org/10.2307/3236223

Varella TL, Rossi AAB, Souza MDA, Silveira, GFS, Cochev JS, Toledo JJ, Silva CJ (2018) Estrutura populacional e distribuição espacial de Theobroma speciosum Willd. Ex Spreng no norte do estado de Mato Grosso. Ciência Florestal, 28(1):115-126. doi: 10.5902/1980509831589

YuenJQ, Fung T, Ziegler AD (2017) Carbon stocks in bamboo ecosystems worldwide: Estimates and uncertainties. Forest Ecology and Management, 393:113-138. doi:10.1016/j.foreco.2017.01.017 